\title{
LncRNA MALAT1 potentiates autophagy-associated cisplatin resistance by regulating the microRNA-30b/autophagy-related gene 5 axis in gastric cancer
}

\author{
ZHIWEN XI ${ }^{1}$, JINCHUN SI ${ }^{2}$ and JUN NAN ${ }^{1}$ \\ ${ }^{1}$ Department of Gastroenterology, The First People's Hospital of Shangqiu; \\ ${ }^{2}$ Department of Surgical Teaching and Research, Shangqiu Medical College, Shangqiu, Henan 476100, P.R. China
}

Received April 17, 2018; Accepted October 2, 2018

DOI: 10.3892/ijo.2018.4609

\begin{abstract}
Gastric cancer (GC) is the fourth most common type of cancer worldwide and chemoresistance is a major obstacle to successful GC treatment. In the present study, reverse transcription-quantitative polymerase chain reaction analysis was used to measure the expression of metastasis-associated lung adenocarcinoma transcript 1 (MALAT1) and microRNA (miR)-30b. Western blot analysis was conducted to detect the protein expression of autophagy-related gene 5 (ATG5), p62 and LC3 (LC3-I and LC3-II). Cell viability and half maximal inhibitory concentration were determined by the Cell Counting Kit- 8 assay. The green fluorescent protein (GFP)-LC3-positive cell percentage was determined by the GFP-LC3 puncta experiment. Luciferase reporter and RNA immunoprecipitation assays were used to explore the molecular associations among MALAT1, miR-30b and ATG5. MALAT1 was found to be highly expressed in CDDP-resistant AGS(AGS/CDDP) cells and CDDP-resistant HGC-27 (HGC-27/CDDP) cells. Cell viability was markedly increased in MALAT1-overexpressing AGS/CDDP cells, but was notably reduced in MALAT1-depleted HGC-27/CDDP cells. Moreover, MALAT1 potentiated CDDP resistance by facilitating autophagy in AGS/CDDP and HGC-27/CDDP cells. Further investigations demonstrated that MALAT1 inhibited miR-30b expression by direct interaction. Moreover, miR-30b abolished MALAT1-induced CDDP resistance by inhibiting autophagy in AGS/CDDP and HGC-27/CDDP cells. Furthermore, ATG5 was found to be a target of miR-30b. miR-30b weakened resistance to CDDP by inhibiting autophagy in AGS/CDDP and HGC-27/CDDP cells, while
\end{abstract}

Correspondence to: Dr Zhiwen Xi, Department of Gastroenterology, The First People's Hospital of Shangqiu, 292 South Kaixuan Rd, Suiyang, Shangqiu, Henan 476100, P.R. China

E-mail: hnslyzgyy2018@126.com

Key words: gastric cancer, microRNA-30b, long non-coding RNA metastasis-associated lung adenocarcinoma transcript 1, autophagy-related gene 5, autophagy, cisplatin this effect was abrogated by increased ATG5 expression. Additionally, MALAT1 sequestered miR-30b from ATG5 to increase ATG5 expression in AGS/CDDP and HGC-27/CDDP cells. Therefore, MALAT1 potentiated autophagy-related CDDP resistance through suppressing the miR-30b/ATG5 axis in AGS/CDDP and HGC-27/CDDP cells, indicating that it may represent a promising target for the reversal of chemoresistance in GC.

\section{Introduction}

Gastric cancer (GC) is currently the fourth most common type of cancer, with the second highest mortality rate worldwide $(1,2)$. It is estimated that $\sim 1,000,000$ new GC cases are diagnosed and $\sim 738,000$ patients succumb to GC annually worldwide (3). Despite the significant advances in the diagnosis, prevention and treatment of GC, its prognosis remains poor, with an overall 5-year survival rate of $\sim 20 \%$ (2).

Systemic chemotherapy is a relatively effective therapeutic approach to patients with advanced and relapsed GC, which account for $80-90 \%$ of all GC patients (4). Innate or acquired chemoresistance represents a major challenge for the treatment of GC $(5,6)$. Cisplatin (CDDP), a known antitumor drug, has been widely used for the treatment of multiple malignancies, such as ovarian and lung cancer (7). Furthermore, CDDP in combination with other drugs (e.g., cetuximab, capecitabine, trastuzumab and sunitinib) has been applied to clinical trials of GC (8). Autophagy, a ‘self-digestion' process, degrades and catabolizes unnecessary/excessive proteins and aged/damaged intracellular organelles to maintain/restore metabolic homeostasis (9). Prior studies also demonstrated that autophagy plays a potential oncogenic or tumor-suppressive role in the development of GC (10). Furthermore, autophagy was found to be closely associated with drug resistance in cancer therapy $(11,12)$. Additionally, CDDP treatment has been shown to improve autophagic activity in certain tumors (e.g., cervical, breast, liver and anaplastic thyroid cancer) $(13,14)$.

Long non-coding RNAs (lncRNAs), a group of transcripts longer than 200 nucleotides (nt) without protein-coding potential, have been identified as positive or negative regulators of autophagy and chemoresistance in cancer $(15,16)$. LncRNA metastasis-associated lung adenocarcinoma 
transcript 1 (MALAT1), also referred to as nuclear-enriched abundant transcript 2 (NEAT2), has been reported to be an oncogene in several cancers, including lung cancer and GC $(17,18)$. Moreover, earlier studies revealed that MALAT1 may enhance chemoresistance of cancer cells by promoting autophagy $(19,20)$.

The aim of the present study was to elucidate the role and underlying molecular mechanisms of MALAT1 in the resistance of GC cells to CDDP.

\section{Materials and methods}

Cell culture. The human GC cell line AGS was obtained from American Type Culture Collection (ATCC, Manassas, VA, USA). The human GC cell line HGC-27 and the 293T cell line were purchased from the cell bank of the Chinese Academy of Sciences (Shanghai, China). AGS cells were maintained in F-12K medium (ATCC) supplemented with $10 \%$ fetal bovine serum (FBS; Invitrogen; Thermo Fisher Scientific, Inc., Carlsbad, CA, USA). HGC-27 cells were cultured in RPMI-1640 medium (Invitrogen; Thermo Fisher Scientific, Inc.) containing $1.5 \mathrm{~g} / 1 \mathrm{NaHCO}_{3}$ (Sigma-Aldrich; Merck KGaA, St. Louis, MO, USA), 2.5 g/l glucose (Sigma-Aldrich; Merck KGaA), 0.11 g/l sodium pyruvate (Sigma-Aldrich; Merck KGaA) and 20\% FBS (Invitrogen; Thermo Fisher Scientific, Inc.). 293T cells were cultured in Dulbecco's modified Eagle's medium (Invitrogen; Thermo Fisher Scientific, Inc.) containing 10\% FBS (Invitrogen; Thermo Fisher Scientific, Inc.). HGC-27 and AGS cells were continuously exposed to gradually increasing concentrations of CDDP $(0.5-10 \mu \mathrm{g} / \mathrm{ml})$ for $>6$ months to establish a CDDP-resistant HGC-27 cell line (HGC-27/CDDP) and a CDDP-resistant AGS (AGS/CDDP) cell line.

Reagents and cell transfection. miR-30b mimic and its scramble control (miR-NC), miR-30b inhibitor and its negative control (anti-miR-30b), small interfering RNA (siRNA) targeting MALAT1 (si-MALAT1) and its negative control (si-NC), were synthesized by GenePharma (Shanghai, China). Full-length sequences of MALAT1 or autophagy-related gene 5 (ATG5) were constructed into a pcDNA3.1 vector (Invitrogen; Thermo Fisher Scientific, Inc.) to generate pcDNA-MALAT1 (MALAT1) or pcDNA-ATG5 (ATG5) overexpression plasmid, respectively. All plasmids or oligonucleotides were transfected into GC cells by Lipofectamine 2000 reagent (Invitrogen; Thermo Fisher Scientific Inc.) according to the instructions of the manufacturer. CDDP was purchased from MedChem Express Co., Ltd. (Monmouth Junction, NJ, USA).

Reverse transcription-quantitative polymerase chain reaction (RT-qPCR) assay. Total RNA (mRNAs and microRNAs) was isolated from GC or CDDP-resistant GC cells using TRIzol reagent (Invitrogen; Thermo Fisher Scientific, Inc.) and quantified by NanoDrop ND 1000 (NanoDrop Technologies, Wilmington, DE, USA). The analysis of miR-30b expression was performed using TaqMan ${ }^{\circledR}$ MicroRNA Real-Time PCR Assay reagents and primers (Applied Biosystems; Thermo Fisher Scientific, Inc., Foster City, CA, USA) with RNU6B (primers also purchased from Applied Biosystems; Thermo Fisher Scientific, Inc.) as an endogenous control. For MALAT1 expression analysis, RNAs were reversely transcribed into first-strand cDNA using the High Capacity cDNA Reverse Transcription kit (Applied Biosystems; Thermo Fisher Scientific, Inc.) and random primers, followed by qPCR detection by SYBR ${ }^{\circledR}$ Premix Ex Taq ${ }^{\mathrm{TM}}$ reagent (Takara, Otsu, Japan) and specific quantitative primers (for MALAT1 or GAPDH). GAPDH was employed to normalize the expression of MALAT1. The primers for MALAT1 or GAPDH were as follows: MALAT1, 5'-CTT AAGCGCAGCGCCATTTT-3' (forward) and 5'-CCTCCA AACCCCAAGACCAA-3' ( $\mathrm{reverse}$ ); G A P D H, 5 ' - C A A C T C CCTCAAGATTGTCAGCAA-3' (forward) and 5'-GGCATG GACTGTGGTCATGA-3' (reverse).

Cell Counting Kit-8 (CCK-8) assay. For the determination of half maximal inhibitory concentration $\left(\mathrm{IC}_{50}\right)$, cells $\left(10^{4} \mathrm{cells} /\right.$ well $)$ were seeded into 96 -well plates and then treated with varying concentrations of CDDP $(0-25 \mu \mathrm{g} / \mathrm{ml})$ for $48 \mathrm{~h}$. For the detection of drug sensitivity, untransfected or transfected cells were treated with CDDP $(5 \mu \mathrm{g} / \mathrm{ml})$ for $48 \mathrm{~h}$. Next, cell viability was determined by the CCK-8 assay kit (Dojindo Molecular Technologies, Rockville, MD, USA) following the manufacturer's instructions. Briefly, $10 \mu 1$ of CCK-8 solution was added into each well of the 96-well plates. After $3 \mathrm{~h}$ of incubation, cell absorbance was examined at $450 \mathrm{~nm}$.

Western blot assay. Total proteins were extracted using RIPA buffer (Beyotime Institute of Biotechnology, Shanghai, China) and quantified using Pierce BCA Protein Assay kit (Thermo Fisher Scientific, Rockford, IL, USA). Subsequently, equal amounts of protein $(50 \mu \mathrm{g})$ were separated by $12 \%$ sodium dodecyl sulfate-polyacrylamide gel electrophoresis and electrophoretically transferred onto nitrocellulose membranes (Pall, New York, NY, USA). After blocking with 5\% skimmed milk for $1 \mathrm{~h}$ at room temperature, the membranes were probed overnight at $4^{\circ} \mathrm{C}$ with rabbit monoclonal antibody against ATG5 (ab108327, 1:2,000 dilution; Abcam, Cambridge, UK), mouse monoclonal antibody against p62 (ab56416, 1:1,000 dilution; Abcam), rabbit polyclonal antibody against LC3B (ab51520, 1:3,000 dilution; Abcam, containing LC3-I and LC3-II), or rabbit polyclonal antibody against $\beta$-actin (ab8227, 1:2,000 dilution; Abcam). Next, the membranes were incubated with horseradish peroxidase-conjugated goat anti-rabbit (ab6721, 1:5,000 dilution; Abcam) or goat anti-mouse (ab6789, 1:5,000 dilution; Abcam) secondary antibody for $1 \mathrm{~h}$ at room temperature. Finally, protein signals were detected by Pierce $^{\mathrm{TM}}$ ECL Western Blotting Substrate (Thermo Fisher Scientific, Inc.) and analyzed by Quantity One software (Bio-Rad Laboratories, Hercules, CA, USA).

Luciferase assay. Partial sequences of MALAT1 or ATG5 3'-untranslated region containing predicted miR-30b-binding sites were constructed into a psiCHECK-2 luciferase vector (Promega Corporation, Madison, WI, USA) to generate MALAT1 wild-type (WT) or ATG5 WT luciferase reporter, respectively. Also, MALAT1 mutant (MUT) or ATG5 MUT luciferase reporter containing mutant miR-30b-binding sites were also produced using GeneArt ${ }^{\mathrm{TM}}$ Site-Directed Mutagenesis System (Invitrogen; Thermo Fisher Scientific, 

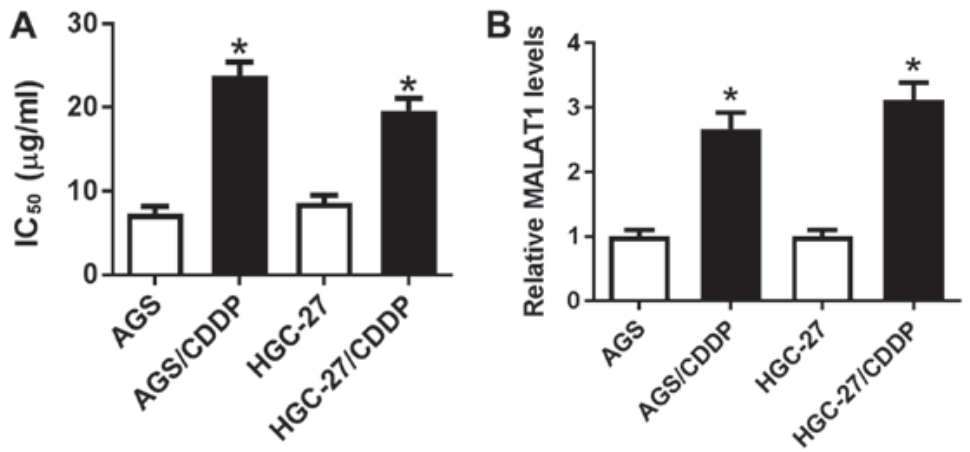

Figure 1. MALAT1 was highly expressed in CDDP-resistant GC cells. (A) AGS, AGS/CDDP, HGC-27 and HGC-27/CDDP cells were exposed with varying concentrations of CDDP (ranging from $0-25 \mu \mathrm{g} / \mathrm{ml}$ ) for $48 \mathrm{~h}$, followed by the determination of $\mathrm{IC}_{50}$ value via CCK-8 assay. (B) Expression patterns of MALAT1 in AGS, AGS/CDDP, HGC-27 and HGC-27/CDDP cells were measured using RT-qPCR analysis. "P<0.05. MALAT1, metastasis-associated lung adenocarcinoma transcript 1; CDDP, cisplatin; RT-qPCR, reverse transcription-quantitative polymerase chain reaction; CCK-8, Cell Counting Kit-8.

Inc.). Then, the constructed luciferase reporters were transfected into $293 \mathrm{~T}$ cells in combination with mimics or plasmids. At $48 \mathrm{~h}$ after transfection, luciferase activities were determined using a dual luciferase reporter assay kit (Promega Corporation).

RNA immunoprecipitation (RIP) assay. RIP assay was performed using Magna RIP ${ }^{\mathrm{TM}}$ RNA-Binding Protein Immunoprecipitation kit (EMD Millipore, Bedford, MA, USA) and primary antibody against IgG (EMD Millipore) or Argonaute 2 (Ago2; EMD Millipore) according to the instructions of the manufacturer.

GFP-LC3 puncta experiment. GC cells and CDDP-resistant GC cells were transfected with pSelect-GFP-hLC3 plasmid (InvivoGen, San Diego, CA, USA) and other plasmids or oligomers (mimics, inhibitors or siRNAs), followed by treatment with CDDP $(5 \mu \mathrm{g} / \mathrm{ml})$ for $48 \mathrm{~h}$. The cells were then fixed using $4 \%$ paraformaldehyde for $20 \mathrm{~min}$ at $4{ }^{\circ} \mathrm{C}$ and stained using DAPI solution $(1 \mu \mathrm{g} / \mathrm{ml}$; Beyotime Institute of Biotechnology) for $15 \mathrm{~min}$. Finally, the cells were visualized using a confocal laser scanning microscope (TCS-TIV; Leica, Nussloch, Germany). The percentage of GFP-LC3-positive cells containing $>20$ puncta was counted in 50 randomly selected fields.

Statistical analysis. Data were obtained from at least 3 independent experiments with the results expressed as mean \pm standard deviation. Student's t-test was used to compare the difference between two groups, and one-way analysis of variance followed by Tukey's post-host test was employed to evaluate the differences among multiple $(>2)$ groups. In all cases, $\mathrm{P}<0.05$ indicated that the difference was statistically significant.

\section{Results}

MALAT1 is highly expressed in CDDP-resistant GC cells. First, CDDP-resistant GC cell lines (AGS/CDDP and HGC-27/CDDP) were established to explore whether MALAT1 was associated with CDDP resistance in GC. As shown in Fig. 1A, the $\mathrm{IC}_{50}$ of CDDP was markedly increased in AGS/CDDP and HGC-27/CDDP cells when compared with that in the corresponding parental cell lines (AGS and HGC-27, respectively), indicating that CDDP-resistant GC cell lines were successfully established. Then, it was further demonstrated that MALAT1 expression was notably upregulated in AGS/CDDP and HGC-27/CDDP cell lines relative to the respective parental cell lines (Fig. 1B), indicating that MALAT1 may be correlated with CDDP resistance in GC.

MALAT1 potentiates CDDP resistance by improving autophagic activity in CDDP-resistant GC cell lines. RT-qPCR analysis further demonstrated that the level of MALAT1 was notably increased in AGS/CDDP cells transfected with MALAT1 overexpression plasmid, but was strikingly reduced in HGC-27/CDDP cells transfected with si-MALAT1 (Fig. 2A). Therefore, MALAT1 overexpression plasmid and si-MALAT1 were used for the following gain-of-function and loss-of-function assays, respectively. Moreover, MALAT1 overexpression induced an obvious increase in AGS/CDDP cell viability (Fig. 2B). Conversely, the viability of MALAT1-silenced HGC-27/CDDP cells was markedly reduced (Fig. 2B). The CCK-8 assay further demonstrated that AGS/CDDP and HGC-27/CDDP cells were more resistant to CDDP compared with their respective parental cells (Fig. 2C). Moreover, ectopic expression of MALAT1 markedly enhanced the resistance of AGS/CDDP cells to CDDP (Fig. 2C). Conversely, MALAT1 knockdown weakened CDDP resistance of HGC-27/CDDP cells (Fig. 2C). Previous studies indicated that MALAT1 improved drug resistance of cancer cells by promoting autophagy $(19,20)$. Hence, the effect of MALAT1 on autophagy was further investigated. The results revealed that cell autophagic activity was markedly increased in CDDP-resistant GC cell lines, as evidenced by an increased percentage of GFP-LC3-positive cells (Fig. 2D), the LC3-II/LC3-I protein ratio was increased (Fig. 2E) and p62 protein expression was reduced (Fig. 2E)in AGS/CDDP and HGC-27/CDDP cells relative to their respective parental cells. Moreover, MALAT1 overexpression facilitated cell autophagy in AGS/CDDP cells, while MALAT1 knockdown suppressed autophagy in HGC-27/CDDP cells (Fig. 2D and E). In summary, these results demonstrated that MALAT1 potentiated CDDP resistance by promoting autophagy in CDDP-resistant GC cells. 

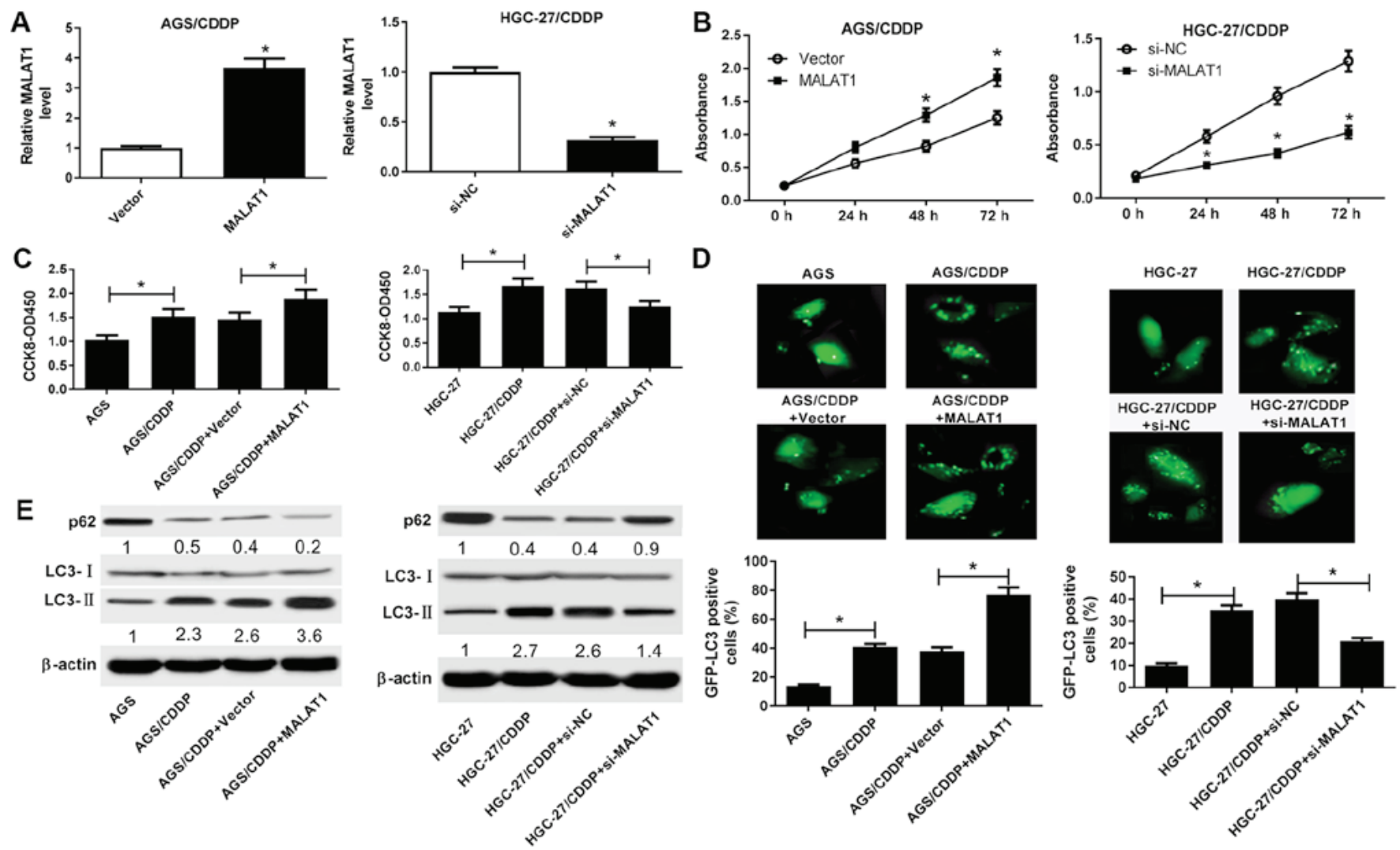

Figure 2. (A-E) MALAT1 potentiated CDDP resistance by promoting autophagy in CDDP-resistant GC cell lines. (A and B) AGS/CDDP cells were transfected with pcDNA3.1 vector or MALAT1 overexpression plasmid, and HGC-27/CDDP cells were transfected with si-NC or si-MALAT1. At $48 \mathrm{~h}$ a post-transfection, the MALAT1 level was determined by RT-qPCR analysis and cell viability was measured by the CCK- 8 assay. (C and E) AGS/CDDP cells were transfected with pcDNA3.1 vector or MALAT1 overexpression plasmid, and HGC-27/CDDP cells were transfected with si-NC or si-MALAT1. At $24 \mathrm{~h}$ post-transfection, transfected or untransfected cells were treated with CDDP $(5 \mu \mathrm{g} / \mathrm{ml})$ for another $48 \mathrm{~h}$. Then, (C) cell viability was determined by the CCK-8 assay and (E) the protein expression of p62, LC3-I and LC3-II was measured by western blotting. (D) The cells in the AGS, AGS/CDDP, AGS/CDDP + vector and AGS/CDDP + MALAT1 groups were transfected with pSelect-GFP-hLC3 plasmid, pSelect-GFP-hLC3 plasmid, pSelect-GFP-hLC3 plasmid + pcDNA3.1 vector and pSelect-GFP-hLC3 plasmid + MALAT1, respectively. At $24 \mathrm{~h}$ post-transfection, the cells were treated with CDDP $(5 \mu \mathrm{g} / \mathrm{ml})$ for another $48 \mathrm{~h}$, followed by detection of the percentage of GFP-LC3-positive cells. "P<0.05. MALAT1, metastasis-associated lung adenocarcinoma transcript 1; CDDP, cisplatin; GC, gastric cancer; RT-qPCR, reverse transcription-quantitative polymerase chain reaction; CCK-8, Cell Counting Kit-8; GFP, green fluorescent protein.

MALAT1 inhibits miR-30b expression by direct interaction. Accumulating evidence supports the hypothesis that lncRNAs may act as competing endogenous RNAs (ceRNAs) to regulate the expression of miRNAs and miRNA target genes in GC (21). Hence, bioinformatics analysis was performed by miRcode online website to identify miRNAs that may interact with MALAT1. Among candidate miRNAs, miR-30b was selected due to its critical role in GC progression and autophagy (22-24) (Fig. 3A). Subsequent luciferase assay demonstrated that miR-30b overexpression markedly reduced luciferase activity of the wild-type MALAT1 reporter, but did not affect luciferase activity of the mutant MALAT1 reporter, suggesting that MALAT1 may interact with miR-30b by putative binding sites (Fig. 3B). To further investigate the spatial interaction between MALAT1 and miR-30b, RIP assay was performed in AGS/CDDP cell lysates using an antibody against Ago2, which is an essential component of the RNA-induced silencing complex (RISC). The results revealed that miR-30b and MALAT1 were substantially enriched in Ago2 immunoprecipitation complexes (Fig. 3C), indicating that MALAT1 was able to spatially interact with miR-30b. RT-qPCR assay further demonstrated that miR-30b expression was notably downregulated in MALAT1-overexpressing
AGS/CDDP cells, but was markedly upregulated in MALAT1-silenced AGS/CDDP cells (Fig. 3D). In summary, these results indicate that MALAT1 inhibits miR-30b expression by direct interaction.

miR-30b abolishes MALAT1-induced CDDP resistance by inhibiting autophagy in CDDP-resistant GC cell lines. Next, restoration experiments were performed to determine whether MALAT1 enhanced CDDP resistance by regulating miR-30b expression in CDDP-resistant GC cells. First, it was demonstrated that the introduction of miR-30b mimic markedly ablated the inhibitory effect of MALAT1 on miR-30b expression in AGS/CDDP cells. Conversely, the transfection of miR-30b inhibitor notably abolished si-MALAT1-induced miR-30b upregulation in HGC-27/CDDP cells (Fig. 4A). Functional analyses revealed that miR-30b overexpression effectively reduced MALAT1-mediated CDDP resistance in AGS/CDDP cells (Fig. 4B). By contrast, the resistance of HGC-27/CDDP cells to CDDP was notably enhanced in si-MALAT1-transfected HGC-27/CDDP cells following miR-30b downregulation (Fig. 4B). Therefore, miR-30b depletion abrogated the suppressive effect of MALAT1 knockdown on CDDP resistance in HGC-27/CDDP cells. 

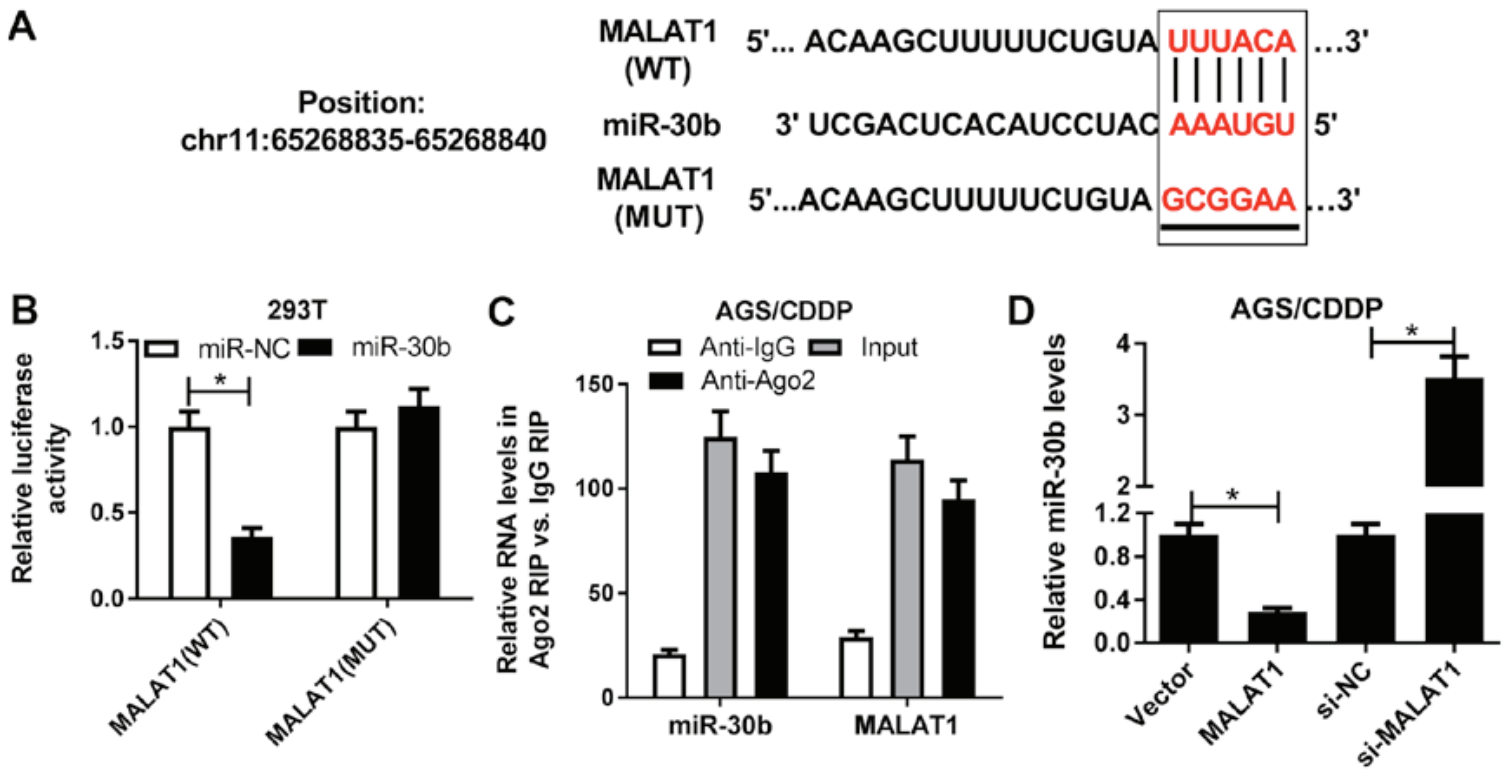

Figure 3. MALAT1 inhibited miR-30b expression by direct interaction. (A) Predicted interaction sites between MALAT1 and miR-30b by miRcode online website, and MUT sites in MALAT1 reporter. (B) 293T cells were co-transfected with MALAT1 WT or MALAT1 MUT reporter and miR-NC or miR-30b. At $48 \mathrm{~h}$ post-transfection, luciferase activity was detected by luciferase assay. (C) RIP assay was performed in AGS/CDDP cell lysates using the antibody against Ago2 or IgG. Then, the expression of miR-30b and MALAT1 in Ago2 or IgG immunoprecipitation complexes was measured via RT-qPCR assay. (D) AGS/CDDP cells were transfected with pcDNA3.1 vector, MALAT1, si-NC or si-MALAT1, followed by the examination of miR-30b level using RT-qPCR assay at $48 \mathrm{~h}$ post-transfection. ${ }^{*} \mathrm{P}<0.05$. MUT, mutant; WT, wild-type; MALAT1, metastasis-associated lung adenocarcinoma transcript 1 ; RIP, RNA immunoprecipitation; Ago2, Argonaute 2; RT-qPCR, reverse transcription-quantitative polymerase chain reaction.

Rescue assays further demonstrated that the restoration of miR-30b attenuated MALAT1-induced autophagy in AGS/CDDP cells, as evidenced by a reduced GFP-LC3-positive cell percentage (Fig. 4C), decreased the LC3-II/LC3-I protein ratio and increased p62 protein expression (Fig. 4D) in MALAT1-enforced AGS/CDDP cells after the introduction of miR-30b mimic. By contrast, the loss of miR-30b relieved si-MALAT1-mediated autophagy inhibition in HGC-27/CDDP cells (Fig. 4C and D). In summary, these data demonstrated that miR-30b weakened MALAT1-induced CDDP resistance by inhibiting autophagy in CDDP-resistant GC cells.

ATG5 is a target of $m i R-30 b$. The TargetScan online website was then employed to search for targets of miR-30b. Among candidate targets, ATG5 was selected on account of its key roles in autophagy and chemoresistance $(11,25)$ (Fig. 5A). A luciferase assay revealed that the introduction of miR-30b mimic resulted in a marked reduction of luciferase activity of the wild-type ATG5 reporter (Fig. 5B). However, miR-30b expression exerted no effect on luciferase activity of the mutant ATG5 reporter (Fig. 5B). These data indicated that miR-30b may interact with ATG5 by predicted binding sites. Moreover, miR-30b upregulation strikingly inhibited ATG5 expression in AGS/CDDP cells (Fig. 5C). Conversely, miR-30b depletion induced a marked increase of the ATG5 level in AGS/CDDP cells (Fig. 5C). Generally, these results confirmed that ATG5 is a target of miR-30b.

miR-30b-mediated inhibitory effects on CDDP resistance and autophagy are abrogated by ATG5 in CDDP-resistant GCcells. It was next demonstrated that ATG5 overexpression reversed the miR-30b-mediated inhibitory effect on ATG5 expression, and ATG5 silencing weakened anti-miR-30b-induced ATG5 upregulation (Fig. 6A). Functional analyses revealed that miR-30b upregulation resulted in reduced CDDP resistance (Fig. 6B), reduced GFP-LC3-positive cell percentage (Fig. 6C), decreased the LC3-II/LC3-I protein ratio and increased p62 protein expression (Fig. 6D) in AGS/CDDP cells. Therefore, miR-30b overexpression alleviated CDDP resistance and inhibited autophagy in AGS/CDDP cells, while these effects were abolished by increased ATG5 expression (Fig. 6B-D). By contrast, ATG5 knockdown rescued anti-miR-30b-induced CDDP resistance and autophagy in HGC-27/CDDP cells (Fig. 6B-D). In summary, these results revealed that miR-30b reduced $C D D P$ resistance and autophagic activity of AGS/CDDP and HGC-27/CDDP cells via targeting ATG5.

MALAT1 promotes ATG5 expression by acting as a ceRNA of miR-30b in CDDP-resistant GC cell lines. We further investigated whether MALAT1 could act as a ceRNA of miR-30b to increase ATG5 expression in AGS/CDDP and HGC-27/CDDP cells. As shown in Fig. 7A, miR-30b overexpression attenuated luciferase activity of the wild-type ATG5 reporter, while this effect was rescued by increased MALAT1 expression in 293T cells. Western blot assay further revealed that ectopic expression of MALAT1 induced the upregulation of the ATG5 protein expression, while this effect was weakened by increased miR-30b expression in AGS/CDDP cells (Fig. 7B). By contrast, ATG5 expression was decreased in MALAT1-silenced HGC-27/CDDP cells and the downregulation of miR-30b alleviated the inhibitory effect of MALAT1 knockdown on ATG5 expression in HGC-27/CDDP cells (Fig. 7C). In conclusion, these data demonstrated that MALAT1 sequestered miR-30b from ATG5, resulting in the upregulation of ATG5 expression in CDDP-resistant GC cells. 

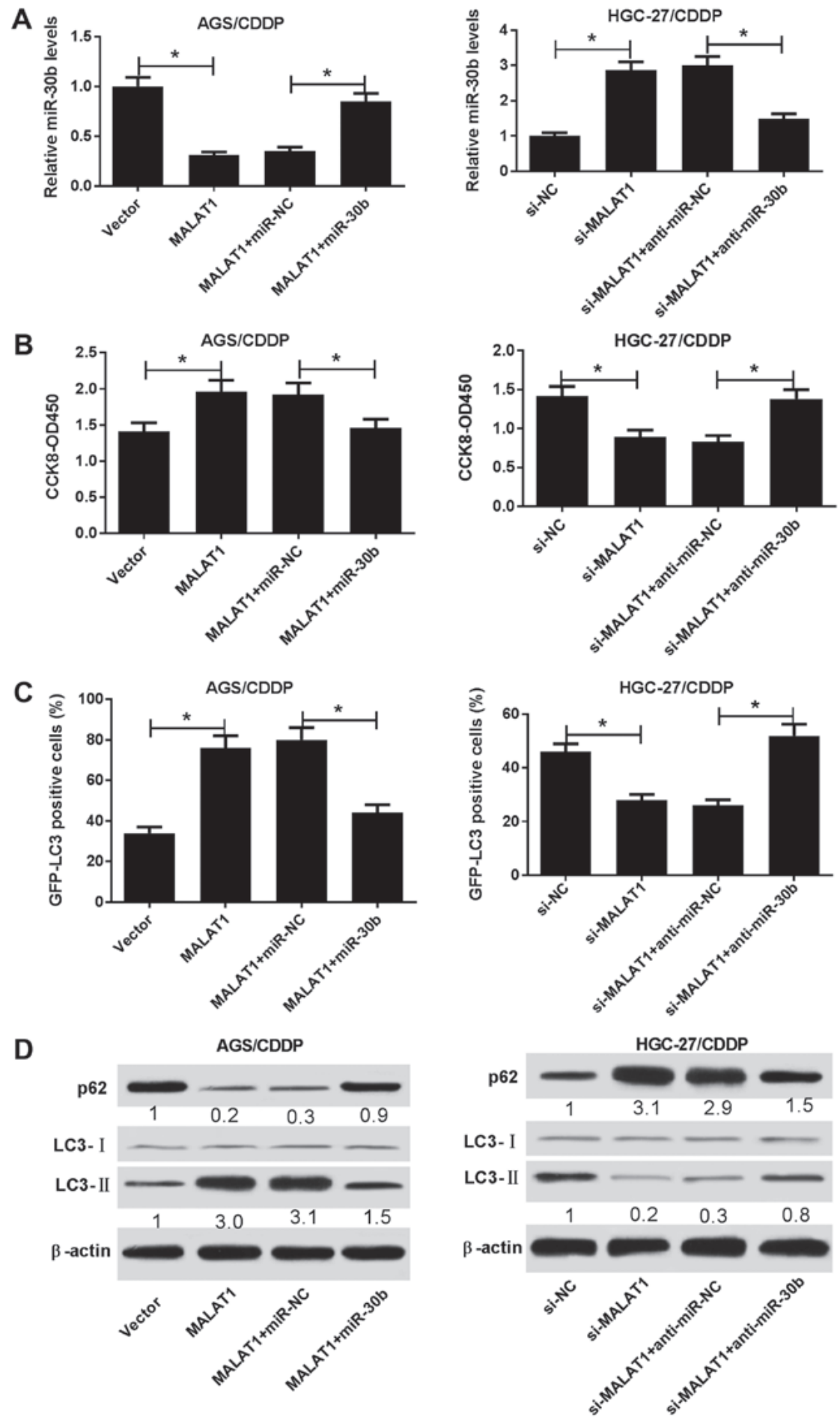

Figure 4. Restoration of miR-30b abolished MALAT1-induced CDDP resistance by inhibiting autophagy in CDDP-resistant GC cell lines. (A-D) AGS/CDDP cells were transfected with pcDNA3.1 vector, MALAT1, MALAT1 + miR-NC, or MALAT1 + miR-30b. HGC-27/CDDP cells were transfected with si-NC, si-MALAT1, si-MALAT1 + anti-miR-NC, or si-MALAT1 + anti-miR-30b. The cells in panel C were co-transfected with pSelect-GFP-hLC3 plasmid. At $24 \mathrm{~h}$ post-transfection, the cells were treated with CDDP $(5 \mu \mathrm{g} / \mathrm{ml})$ for $48 \mathrm{~h}$, followed by the determination of (A) miR-30b level, (B) cell viability, (C) percentage of GFP-LC3-positive cells and (D) protein expression of p62, LC3-I and LC3-II. "P<0.05. ATG5, autophagy-related gene 5; CDDP, cisplatin; GC, gastric cancer; GFP, green fluorescent protein.

\section{Discussion}

Current therapeutic strategies (e.g., surgical resection and adjuvant chemotherapy) are generally not curative for the majority of GC patients, particularly those diagnosed with metastatic and advanced GC, due to various reasons, including innate and acquired chemoresistance (6).
Consequently, it is imperative to elucidate the molecular mechanisms underlying GC chemoresistance in order to design more effective interventions. Autophagy, characterized by the conversion of LC3-I to LC3-II and the formation of autolysosomes (the complex of autophagosomes and lysosomes), plays a dual role (oncogenic or antitumor) in cancer initiation and progression $(26,27)$. Moreover, 
A

Position 707-714 of ATG5 3' UTR

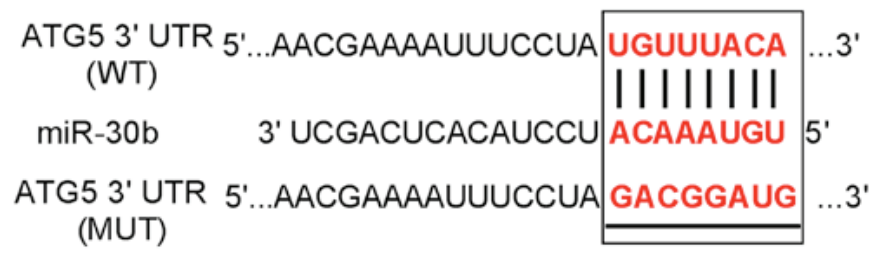

B
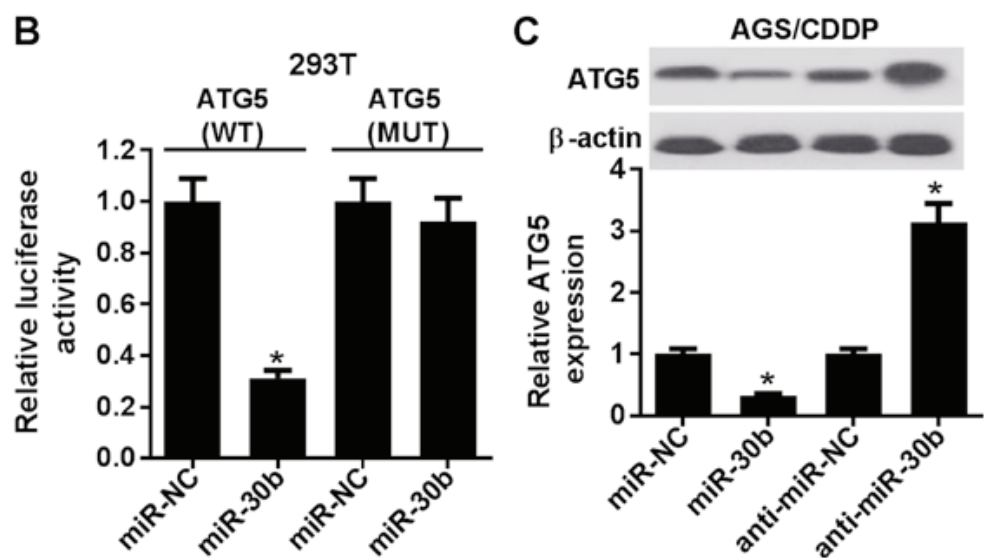

Figure 5. ATG5 was a target of miR-30b. (A) Predicted binding sites between miR-30b and the ATG5 3'-untranslated region by TargetScan online website, and MUT sites in ATG5 reporter. (B) 293T cells were co-transfected with ATG5 WT or ATG5 MUT reporter and miR-NC or miR-30b mimic, followed by the measurement of luciferase activity at $48 \mathrm{~h}$ post-transfection. (C) AGS/CDDP cells were tranfected with miR-NC, miR-30b, anti-miR-NC, or anti-miR-30b. At $48 \mathrm{~h}$ post-transfection, the ATG5 protein level was tested using western blot assay. ${ }^{*} \mathrm{P}<0.05$. ATG5, autophagy-related gene 5; CDDP, cisplatin; MUT, mutant; WT, wild-type.
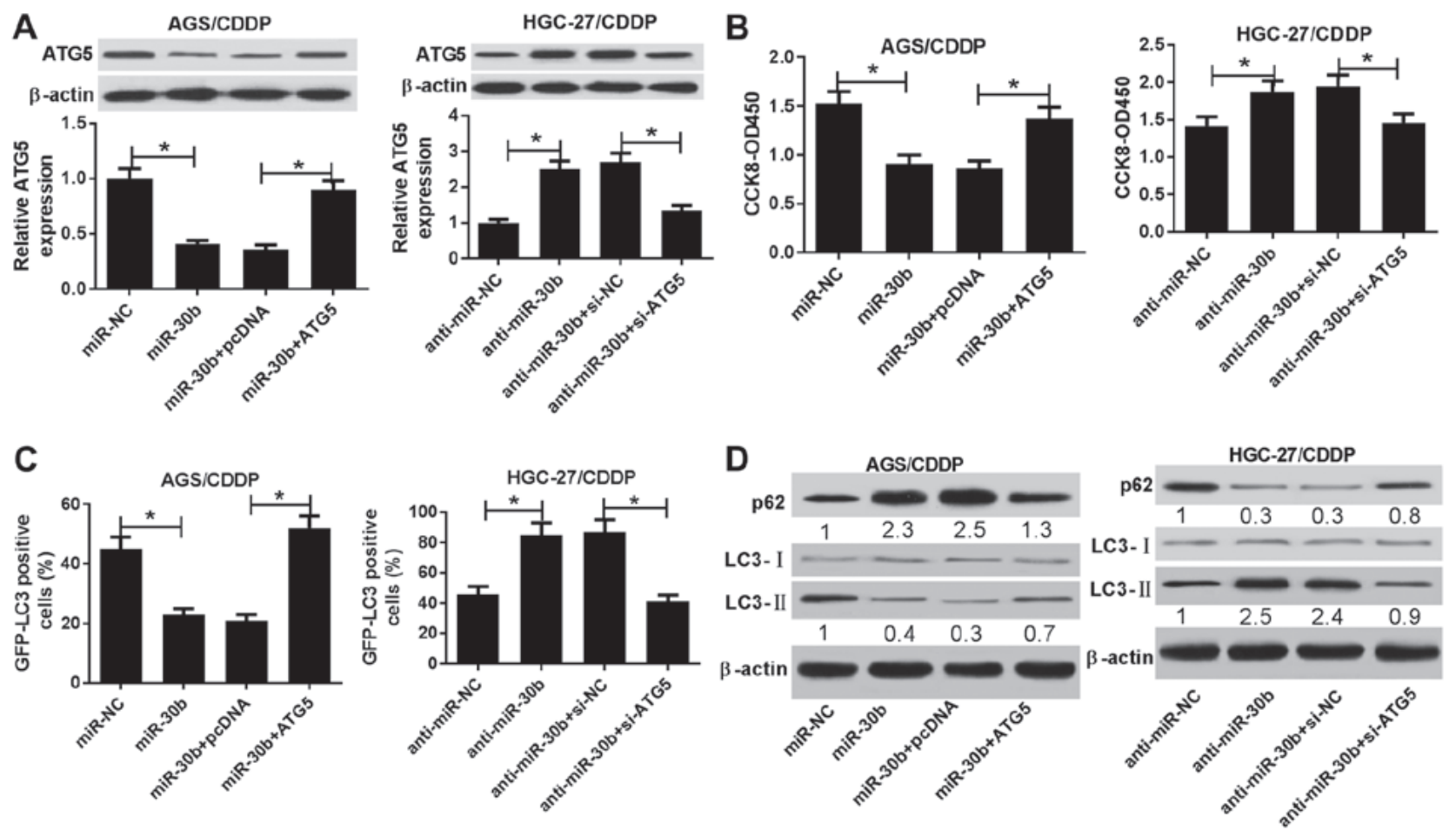

Figure 6. miR-30b reduced CDDP resistance and autophagic activity of AGS/CDDP and HGC-27/CDDP cells via targeting ATG5. (A-D) AGS/CDDP cells were transfected with miR-NC, miR-30b, miR-30b + pcDNA3.1, or miR-30b + ATG5. HGC-27/CDDP cells were transfected with anti-miR-NC, anti-miR-30b, anti-miR-30b + si-NC, or anti-miR-30b + si-ATG5. Also, cells in panel C were co-transfected with pSelect-GFP-hLC3 plasmid. At $24 \mathrm{~h}$ post-transfection, cells were treated with CDDP $(5 \mu \mathrm{g} / \mathrm{ml})$ for $48 \mathrm{~h}$, followed by the determination of (A) ATG5 level, (B) cell viability, (C) percentage of GFP-LC3-positive cells and(D) protein expression of p62, LC3-I and LC3-II. "P<0.05. CDDP, cisplatin; ATG5, autophagy-related gene 5; GFP, green fluorescent protein.

autophagy exerts a dual effect on the therapeutic efficacy of chemotherapy drugs: It may enhance the therapeutic efficacy of drugs, or it may reduce therapeutic efficacy and enhance drug resistance $(11,12)$. 
A

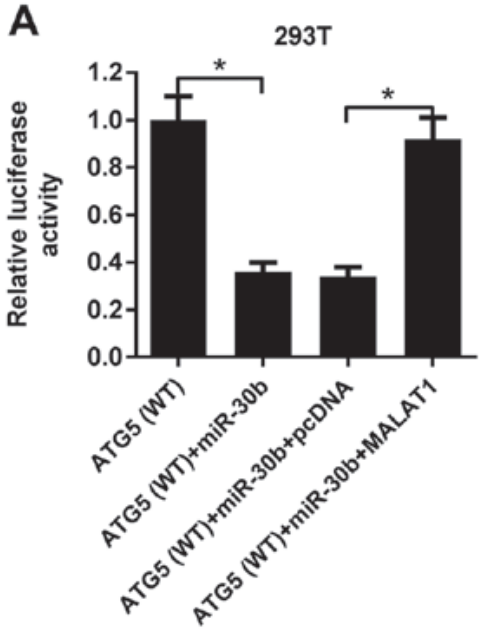

B

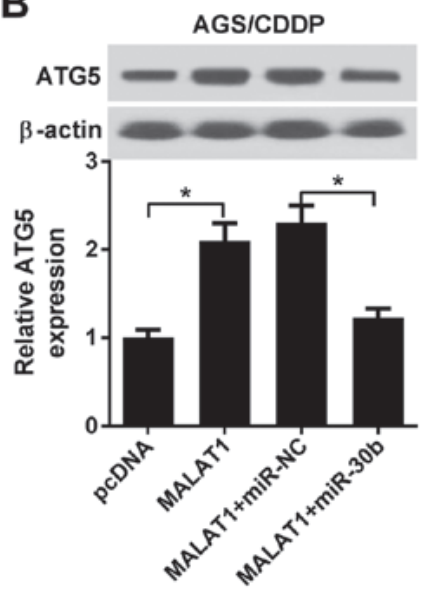

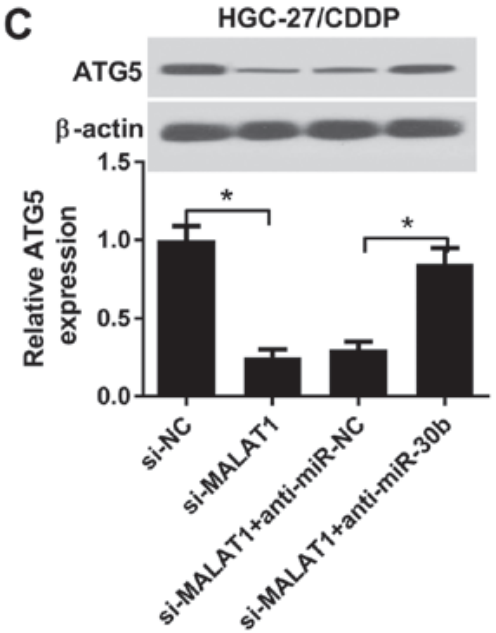

Figure 7. MALAT1 promoted ATG5 expression by acting as a ceRNA of miR-30b in CDDP-resistant GC cell lines. (A) 293T cells were co-transfected with ATG5 WT reporter, ATG5 WT reporter + miR-30b, ATG5 WT reporter + miR-30b + pcDNA3.1 vector, or ATG5 WT reporter +miR-30b + MALAT1, followed by the measurement of luciferase activity at $48 \mathrm{~h}$ post-transfection. (B) AGS/CDDP cells were transfected with pcDNA3.1 vector, MALAT1, MALAT1 + miR-NC, or MALAT1 + miR-30b. Then, ATG5 protein level was detected at $48 \mathrm{~h}$ post-transfection. (C) HGC-27/CDDP cells were transfected with si-NC, si-MALAT1, si-MALAT1 + anti-miR-NC, or si-MALAT1 + anti-miR-30b, followed by measurement of ATG5 protein level at 48 h post-transfection. ${ }^{*} \mathrm{P}<0.05$. MALAT1, metastasis-associated lung adenocarcinoma transcript 1; ATG5, autophagy-related gene 5; ceRNA, competingendogenous RNA; CDDP, cisplatin; WT, wild-type.

Accumulating evidence indicates that miRNAs and lncRNAs are closely correlated with chemoresistance and autophagy in cancer $(15,28,29)$. LncRNA MALAT1 was found to be aberrantly highly expressed in GC, whereas MALAT1 knockdown inhibited GC progression (30-32). Moreover, MALAT1 has been reported to be implicated in chemoresistance and autophagy in certain types of cancer $(19,20)$. For example, Yuan et al demonstrated that the depletion of MALAT1 reduced chemoresistance of multidrug-resistant hepatocellular cancer cells to 5-fluorouracil (5-FU), adriamycin or mitomycin C by inhibiting autophagy (19). Additionally, YiRen et al observed that MALAT1 knockdown weakened the chemoresistance of vincristine (VCR)-resistant GC cells to 5-FU, VCR and CDDP by suppressing autophagy via regulating the miR-23b-3p/ATG12 axis (20).

In the present study, it was demonstrated that MALAT1 expression was notably upregulated in CDDP-resistant GC (AGS/CDDP and HGC-27/CDDP) cells. YiRen et al also reported that MALAT1 was highly expressed in VCR-resistant SGC7901 (SGC7901/VCR) cells compared with parental cells (20). Moreover, cell autophagic activity was improved in CDDP-resistant GC cells compared with parental cells. Increased autophagic activity was also observed in SGC7901/VCR cells compared with that in parental SGC7901 cells (20). Functional analyses revealed that MALAT1 overexpression potentiated CDDP resistance of AGS/CDDP cells by promoting autophagy. Conversely, MALAT1 knockdown reduced the resistance of HGC-27/CDDP cells to CDDP by inhibiting autophagy.

miR-30b is a member of the miR-30 family, which also includes miR-30a, miR-30c, miR-30d and miR-30e (33). It was previously demonstrated that other members of the miR-30 family were implicated in mediating CDDP resistance by regulating autophagy in certain types of cancer. For example, miR-30a overexpression weakened CDDP-induced autophagy, but boosted CDDP-induced apoptosis in cervical cancer
(HeLa) cells (13). Therefore, miR-30a promoted sensitivity of HeLa cells to CDDP by inhibiting autophagy (13). Zhang et al also demonstrated that miR-30d increased the sensitivity of anaplastic thyroid cancer cells to CDDP by reducing autophagic activity (14). miR-30b has been reported to be downregulated in GC and was shown to inhibit the development of GC by targeting plasminogen activator inhibitor-1 (22). Moreover, miR-30b overexpression inhibited high phosphorus (Pi)-induced autophagy by reducing the expression of autophagy-related genes, such as ATG5, in vascular smooth muscle cells (24). Additionally, miR-30b suppressed autophagy to abate hepatic ischemia-reperfusion injury by reducing ATG12-ATG5 conjugates (23). The abovementioned findings indicated that miR-30b may regulate CDDP resistance and autophagy. In the present study, it was further demonstrated that MALAT1 inhibited miR-30b expression by direct interaction in AGS/CDDP cells. Furthermore, restoration assays revealed that miR-30b upregulation abrogated MALAT1-induced CDDP resistance by inhibiting autophagy in CDDP-resistant GC cells.

Accumulating evidence shows that miRNAs may exert their effects by regulating target gene expression (34). Hence, ATG5 as a target of miR-30b was validated by bioinformatics analysis and luciferase assay, and the results were in line with those of previous studies $(35,36)$. ATG5, a key regulator of autophagy (25), has been generally considered as a protective factor of tumor cells against chemotherapy (11). ATG5 expression was markedly upregulated in CDDP-resistant GC cells (SGC7901/CDDP) and the depletion of ATG5 decreased chemoresistance of SGC7901/CDDP cells to CDDP. The findings of the present study revealed that miR-30b weakened autophagy-related CDDP resistance by targeting ATG5 in CDDP-resistant GC cells.

It was further confirmed that MALAT1 may act as a ceRNA of miR-30b to sequester miR-30b from ATG5, resulting in the upregulation of ATG5 expression in CDDP-resistant GC cells. 
However, YiRen et al reported that MALAT1 knockdown exerted no effect on ATG5 mRNA level in SGC7901/VCR cells (20), whereas Li et al demonstrated that MALAT1 knockdown resulted in the increase of ATG5 expression in diffuse large B-cell lymphoma cells (37). It may be hypothesized that this difference among reported results may be due to the different intracellular environments.

Collectively, the findings of the present study revealed that MALAT1 potentiated CDDP resistance by inducing autophagy via regulating the miR-30b/ATG5 axis in CDDP-resistant GC cells, improving our understanding of the role and molecular function of MALAT1 in drug resistance, and indicating potential therapeutic strategies to prevent or reverse drug resistance.

\section{Acknowledgements}

Not applicable.

\section{Funding}

No funding was received.

\section{Availability of data and materials}

All the data sets generated and analyzed in this study are available from the corresponding author on reasonable request.

\section{Authors' contributions}

This study was conceived and designed by ZX and JS. The experiments were carried out by $\mathrm{JN}$ and $\mathrm{ZX}$. The manuscript was prepared by ZX, JS and JN. All the authors have read and approved the final version of this manuscript.

\section{Ethics approval and consent to participate}

Not applicable.

\section{Patient consent for publication}

Not applicable.

\section{Competing interests}

The authors declare that they have no competing interests to disclose.

\section{References}

1. Jemal A, Center MM, DeSantis C and Ward EM: Global patterns of cancer incidence and mortality rates and trends. Cancer Epidemiol Biomarkers Prev 19: 1893-1907, 2010.

2. Karimi P, Islami F, Anandasabapathy S, Freedman ND and Kamangar F: Gastric cancer: Descriptive epidemiology, risk factors, screening, and prevention. Cancer Epidemiol Biomarkers Prev 23: 700-713, 2014.

3. Ferlay J, Shin HR, Bray F, Forman D, Mathers C and Parkin DM: Estimates of worldwide burden of cancer in 2008: GLOBOCAN 2008. Int J Cancer 127: 2893-2917, 2010.

4. Wagner AD, Unverzagt S, Grothe W, Kleber G, Grothey A, Haerting J and Fleig WE: Chemotherapy for advanced gastric cancer. Cochrane Database Syst Rev 3: CD004064, 2010.
5. Charalampakis N, Economopoulou P, Kotsantis I, Tolia M, Schizas D, Liakakos T, Elimova E, Ajani JA and Psyrri A: Medical management of gastric cancer: A 2017 update. Cancer Med 7: 123-133, 2018.

6. Zhang D and Fan D: Multidrug resistance in gastric cancer: Recent research advances and ongoing therapeutic challenges. Expert Rev Anticancer Ther 7: 1369-1378, 2007.

7. Cohen SM and Lippard SJ: Cisplatin: From DNA damage to cancer chemotherapy. Prog Nucleic Acid Res Mol Biol 67: 93-130, 2001.

8. Xu W, Yang Z and Lu N: Molecular targeted therapy for the treatment of gastric cancer. J Exp Clin Cancer Res 35: 1, 2016.

9. Mizushima N and Klionsky DJ: Protein turnover via autophagy: Implications for metabolism. Annu Rev Nutr 27: 19-40, 2007.

10. Qian HR and Yang Y: Functional role of autophagy in gastric cancer. Oncotarget 7: 17641-17651, 2016.

11. Sui X, Chen R, Wang Z, Huang Z, Kong N, Zhang M, Han W, Lou F, Yang J, Zhang Q, et al: Autophagy and chemotherapy resistance: A promising therapeutic target for cancer treatment. Cell Death Dis 4: e838, 2013.

12. Kumar A, Singh UK and Chaudhary A: Targeting autophagy to overcome drug resistance in cancer therapy. Future Med Chem 7: $1535-1542,2015$

13. Zou Z, Wu L, Ding H, Wang Y, Zhang Y, Chen X, Chen X, Zhang CY, Zhang Q and Zen K: MicroRNA-30a sensitizes tumor cells to cis-platinum via suppressing beclin 1-mediated autophagy. J Biol Chem 287: 4148-4156, 2012.

14. Zhang Y, Yang WQ, Zhu H, Qian YY, Zhou L, Ren YJ, Ren XC, Zhang L, Liu XP, Liu CG, et al: Regulation of autophagy by miR-30d impacts sensitivity of anaplastic thyroid carcinoma to cisplatin. Biochem Pharmacol 87: 562-570, 2014.

15. Sun T: Long noncoding RNAs act as regulators of autophagy in cancer. Pharmacol Res 129: 151-155, 2017.

16. Majidinia M and Yousefi B: Long non-coding RNAs in cancer drug resistance development. DNA Repair (Amst) 45: 25-33, 2016.

17. Gutschner T, Hämmerle M and Diederichs S: MALAT1-a paradigm for long noncoding RNA function in cancer. J Mol Med (Berl) 91: 791-801, 2013.

18. Li Y, Wu Z, Yuan J, Sun L, Lin L, Huang N, Bin J, Liao Y and Liao W: Long non-coding RNA MALAT1 promotes gastric cancer tumorigenicity and metastasis by regulating vasculogenic mimicry and angiogenesis. Cancer Lett 395: 31-44, 2017.

19. Yuan P, Cao W, Zang Q, Li G, Guo $X$ and Fan J: The HIF-2 $\alpha$-MALAT1-miR-216b axis regulates multi-drug resistance of hepatocellular carcinoma cells via modulating autophagy. Biochem Biophys Res Commun 478: 1067-1073, 2016.

20. YiRen H, YingCong Y, Sunwu Y, Keqin L, Xiaochun T, Senrui C, Ende C, XiZhou L and Yanfan C: Long noncoding RNA MALAT1 regulates autophagy associated chemoresistance via miR-23b-3p sequestration in gastric cancer. Mol Cancer 16: 174, 2017.

21. Hu Y, Tian H, Xu J and Fang JY: Roles of competing endogenous RNAs in gastric cancer. Brief Funct Genomics 15: 266-273, 2016.

22. Zhu ED, Li N, Li BS, Li W, Zhang WJ, Mao XH, Guo G, Zou QM and Xiao B: miR-30b, down-regulated in gastric cancer, promotes apoptosis and suppresses tumor growth by targeting plasminogen activator inhibitor-1. PLoS One 9: e106049, 2014.

23. Li SP, He JD, Wang Z, Yu Y, Fu SY, Zhang HM, Zhang JJ and Shen ZY: miR-30b inhibits autophagy to alleviate hepatic ischemia-reperfusion injury via decreasing the Atg12-Atg5 conjugate. World J Gastroenterol 22: 4501-4514, 2016.

24. Wang J, Sun YT, Xu TH, Sun W, Tian BY, Sheng ZT, Sun L, Liu LL, Ma JF, Wang LN, et al: MicroRNA-30b Regulates High Phosphorus Level-Induced Autophagy in Vascular Smooth Muscle Cells by Targeting BECN1. Cell Physiol Biochem 42: 530-536, 2017.

25. Klionsky DJ: Autophagy: from phenomenology to molecular understanding in less than a decade. Nat Rev Mol Cell Biol 8: 931-937, 2007.

26. Tanida I, Ueno T and Kominami E: LC3 and Autophagy. Methods Mol Biol 445: 77-88, 2008.

27. White E, Mehnert JM and Chan CS: Autophagy, Metabolism, and Cancer. Clin Cancer Res 21: 5037-5046, 2015.

28. Pan B, Yi J and Song H: MicroRNA-mediated autophagic signaling networks and cancer chemoresistance. Cancer Biother Radiopharm 28: 573-578, 2013.

29. Ayers D and Vandesompele J: Influence of microRNAs and Long Non-Coding RNAs in Cancer Chemoresistance. Genes (Basel) 8: 95, 2017. 
30. Wang J, Su L, Chen X, Li P, Cai Q, Yu B, Liu B, Wu W and Zhu Z: MALAT1 promotes cell proliferation in gastric cancer by recruiting SF2/ASF. Biomed Pharmacother 68: 557-564, 2014

31. Qi Y, Ooi HS, Wu J, Chen J, Zhang X, Tan S, Yu Q, Li YY, Kang Y, Li H, et al: MALAT1 long ncRNA promotes gastric cancer metastasis by suppressing PCDH10. Oncotarget 7: 12693-12703, 2016.

32. Xia H, Chen Q, Chen Y, Ge X, Leng W, Tang Q, Ren M, Chen L, Yuan D, Zhang Y, et al: The IncRNA MALAT1 is a novel biomarker for gastric cancer metastasis. Oncotarget 7: 56209-56218, 2016.

33. Ouzounova M, Vuong T, Ancey PB, Ferrand M, Durand G, Le-Calvez Kelm F, Croce C, Matar C, Herceg Z and Hernandez-Vargas H: MicroRNA miR-30 family regulates non-attachment growth of breast cancer cells. BMC Genomics 14 $139,2013$.
34. Bartel DP: MicroRNAs: Genomics, biogenesis, mechanism, and function. Cell 116: 281-297, 2004.

35. Liu Z, Wei X, Zhang A, Li C, Bai J and Dong J: Long non-coding RNA HNF1A-AS1 functioned as an oncogene and autophagy promoter in hepatocellular carcinoma through sponging hsa-miR-30b-5p. Biochem Biophys Res Commun 473: 1268-1275, 2016.

36. Chen Z, Jin T and Lu Y: AntimiR-30b Inhibits TNF- $\alpha$ Mediated Apoptosis and Attenuated Cartilage Degradation through Enhancing Autophagy. Cell Physiol Biochem 40: 883-894, 2016.

37. Li LJ, Chai Y, Guo XJ, Chu SL and Zhang LS: The effects of the long non-coding RNA MALAT-1 regulated autophagy-related signaling pathway on chemotherapy resistance in diffuse large B-cell lymphoma. Biomed Pharmacother 89: 939-948, 2017. 